\section{OUTCOME AFTER INFLICTED TRAUMATIC BRAIN INJURY IN SHAKEN BABY SYNDROME: NEUROSURGICAL APPROACH}

doi:10.1136/archdischild-2012-302724.1105

F Menegazzo, M Bua, M Rosa Rizzotto, E Sgaravatti, P Facchin, R Faggin. Department of Pediatrics, Child Abuse Unit, University of Padova, Padova, Italy

Background and Aims Shaken Baby Syndrome (SBS) is a severe form of child abuse caused by violent shaking leading to severe head injuries, causing mild-severe long-term disabilities and death. The study aims to explore medium/long-term consequences of SBS comparing children undergoing neurosurgery with hematoma evacuation/cranioplasty with those with hematoma evacuation or no surgery.

Methods A cohort of 21 children with SBS, admitted to Pediatric Department/Padua Hospital (2003-2011), was followed-up. Each clinical record was reviewed collecting information on onset, acute course, ophthalmologic examinations, neuroimaging, treatment and procedures, family history and social background. Cases were followed-up at 3, 6 and 12 months after trauma, every year. The assessment included fundus evaluation and visual function, neuroradiological exams (MRI).

Results 21 cases were reviewed (M:F=2:1.1), 12 foreigners, 9 italians. Mean age at onset: 5.7 months (range 1.2-18). Mean age at last follow-up evaluation: 30.4 months (range 5-82). Mean follow-up duration: 24.6 months (range 1-73.5). In acute phase 8/19 underwent neurosurgical intervention and $1 / 21$ underwent eye surgery. 20/21 showed retinal hemorrhages, 21/21 cerebral hemorrhages, $11 / 21$ cerebellar hemorrhages, $5 / 10$ spinal subdural hematoma, 4/21 skull fractures, $6 / 21$ other body region fractures. At last follow-up evaluation resulted: $2 / 21$ hemiplegia, $1 / 21$ paraplegia, $1 / 21$ tetraplegia, $2 / 21$ cortical visual impairment, $3 / 21$ visual field deficits, 4/21 strabismus. 10/21 underwent cognitive and behavioural assessment demostrating in 6 cases global delay and in 4 cases delay in specific functions (locomotor, eye and hand coordination, performance scale). Surgical procedure's video will be shown.

Conclusions SBS may influence child development and therapeutical surgical approach seems crucial.

\section{IMPACT OF WARMED INHALED GAS FROM THE MECHANICAL VENTILATOR ON ESOPHAGEAL TEMPERATURE DURING WHOLE BODY HYPOTHERMIA FOR HYPOXIC-ISCHEMIC ENCEPHALOPATHY}

doi:10.1136/archdischild-2012-302724.1106

'S Sarkar, ${ }^{2}$ SS Sarkar, ${ }^{3}$ Bhagat, 'R Dechert, 'SM Donn. 'Department of Pediatrics, Division of Neonatal-Perinatal Medicine; ${ }^{2}$ Research Volunteer, Division of NeonatalPerinatal Medicine, University of Michigan Health System; ${ }^{P}$ Pediatrics, St Joseph Mercy Hospital, Ann Arbor, MI, USA

During whole body cooling (WBC), the core temperature is monitored with either an esophageal or a rectal probe. Most infants are usually on mechanical ventilation while receiving hypothermia. As the temperature in the esophagus responds rapidly to changes in the ambient temperature, inhalation of warmed gas from ventilator during hypothermia may lead to overestimation of ventilated patients' actual temperature, causing automated cooling devices to overcool patients well below set temperature targets.

Objective We determined if the esophageal temperature recordings during therapeutic WBC differ between ventilated and nonventilated infants.

Methods Twenty-two consecutively cooled infants had simultaneous esophageal and rectal temperatures recorded every 4 hours during 72 hours of WBC. The later was deemed to be actual core temperature. Other clinical monitoring and treatment during hypothermia were as per established protocol.
Results Fourteen infants received mechanical ventilation throughout cooling. The remaining 8 infants were on ventilator initially but got extubated and were not on ventilator during 32 to 72 hours section of WBC. Esophageal temperatures were significantly higher than simultaneous rectal temperatures ( $p \leq 0.01$ at each time point) for all 22 infants. However, the esophageal temperatures across every 4 hour time points during 32 to 72 hours section of WBC did not differ between the ventilated (n-14), and non-ventilated (n-8) infants. The magnitude (median, IOR) of the difference between esophageal and rectal temperatures were also similar between the 2 groups.

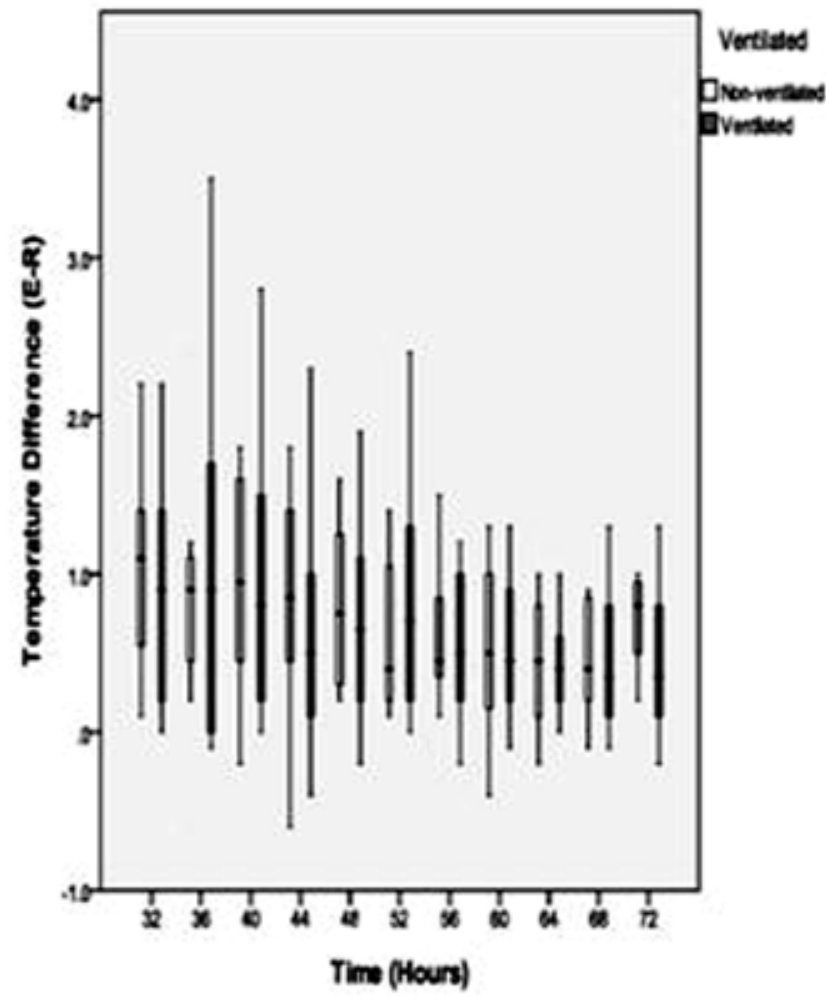

Abstract 1106 Figure 1 Comparison of E-R (in centigrade) between 2 groups

Conclusions Warmed inhaled gas does not interfere with the esophageal temperature during WBC.

\section{CONTINUOUS CARBON DIOXIDE MONITORING USING FEATURES OF NEONATAL ELECTROENCEPHALOGRAPHY}

doi:10.1136/archdischild-2012-302724.1107

${ }^{1} \mathrm{C}$ McKeering, ${ }^{1 P}$ Gaydecki, ${ }^{2} \mathrm{~A}$ Hendrickson, ${ }^{2} \mathrm{C}$ Jennings, $2,3 \mathrm{~S}$ Victor. ${ }^{1}$ School of Electrical and Electronic Engineering, University of Manchester; ${ }^{2}$ Newborn Intensive Care Unit, Central Manchester University Hospitals NHS Foundation Trust; ${ }^{3}$ School of Biomedicine, University of Manchester, Manchester, UK

Background The continuous monitoring of partial pressure of blood carbon dioxide $\left(\mathrm{pCO}_{2}\right)$ in premature babies has proven to be challenging. Spot measurements of $\mathrm{pCO}_{2}$ can be performed by taking a blood sample. However the frequency of such measurements is limited by their invasiveness.

Aim We aim to develop a continuous non-invasive method of predicting $\mathrm{pCO}_{2}$ using features of the preterm electroencephalography (EEG) signal.

Methods A regression model was trained on eight 12 hour EEG recordings that contained 22 blood gas measurements in total. All measurements were obtained from babies born before 28 weeks' gestation and less than 72 hours old. The duration of EEG quiescence 